\title{
Análisis de calidad de potencia en un sistema industrial a partir de mediciones multipunto
}

\author{
J. Jeffer Vera* \\ Francisco Santamaría** \\ Adolfo Jaramillo***
}

\author{
Recibido: 15/09/2016 - Aceptado: 07/09/2017 \\ DOI: 10.22395/rium.v17n32a9
}

\begin{abstract}
Resumen
Este artículo propone una metodología para el análisis de calidad de potencia en instalaciones eléctricas industriales. Inicialmente se realiza una medición multipunto, después se identifican y analizan los fenómenos que más afectan a la industria, luego se modela y ajusta el sistema al comportamiento real, y se proponen y simulan algunas soluciones. Posteriormente, se selecciona un caso de estudio y se realiza el estudio de calidad de potencia a partir de la metodología propuesta y según los parámetros de la norma NTC 5001. Finalmente, se establecen las ventajas del uso de la medición multipunto y el modelado en estudios de calidad de potencia.
\end{abstract}

Palabras clave: armónicos; calidad de potencia; hundimientos de tensión; medición multipunto; modelado; simulación.

\footnotetext{
Ingeniero eléctrico. Universidad Distrital Francisco José de Caldas. Carrera 7 \#40B-53 Piso 7, Bogotá. jlveram@ correo.udistrital.edu.co

** Ingeniero electricista. Ph. D., M. Sc. en Ingeniería. Profesor asociado. Universidad Distrital Francisco José de Caldas. Carrera 7 \#40B-53 Piso 5, Bogotá. fsantamariap@udistrital.edu.co

*** Ingeniero electrónico. Ph. D., M. Sc. en Ingeniería Electrónica, Automática y Telecomunicaciones. Profesor asociado. Universidad Distrital Francisco José de Caldas. Carrera 7 \#40B-53 Piso 5, Bogotá. ajaramillom@ udistrital.edu.co
} 


\title{
Power Quality Analysis in an Industrial System from Multipoint Measurements
}

\begin{abstract}
This paper proposes a methodology for the analysis of power quality in industrial electrical installations. Initially, a multipoint measurement is performed, then the phenomena that most affect the industry are identified and analyzed, then the system is modelled and adjusted to the real behavior, and some solutions are proposed and simulated. Subsequently, a case study is selected and the power quality study is carried out on the basis of the proposed methodology and according to the parameters of the NTC 5001 standard. Finally, the advantages of using multipoint measurement and modeling in power quality studies are established.
\end{abstract}

Keywords: harmonics; power quality; voltage sags; multipoint measurement; modelling; simulation.

\section{Análise de qualidade de potência num sistema industrial a partir de medições multipontos}

\begin{abstract}
Resumo
Este artigo propõe uma metodologia para a análise de qualidade de potência em instalações elétricas industriais. Inicialmente, realiza-se uma medição multiponto; logo, são identificados e analisados os fenômenos que mais afetam a indústria; em seguida, o sistema é modelado e ajustado ao comportamento real, e algumas soluções são propostas e simuladas. Posteriormente, seleciona-se um caso de estudo e realiza-se o estudo de qualidade de potência a partir da metodologia proposta e segundo os parâmetros da norma NTC 5001. Finalmente, estabelecem-se as vantagens do uso da medição multiponto e o modelado em estudos de qualidade de potência.
\end{abstract}

Palavras-chave: harmônicos; qualidade de potência; afundamento de tensão; medição multiponto; modelado; simulação. 


\section{INTRODUCCIÓN}

Hay eventos que pueden ocasionar problemas de calidad de potencia, por lo cual es necesario saber con exactitud su origen y localización, con el fin de disminuir su impacto en el sistema e incluso prevenir, de ser posible, su ocurrencia. El procedimiento regular es instalar un medidor, realizar registros, analizar la información, reinstalar el medidor en varios puntos y realizar nuevos análisis [1, 2]. De esta manera, se puede establecer el comportamiento de los parámetros eléctricos en un punto determinado del sistema, pero no se puede conocer cuál fue su comportamiento global ante un evento específico, lo que dificulta el análisis en sistemas con distribuciones extensas.

Una solución es realizar una medición multipunto mediante el uso de varios equipos registradores sincronizados, instalados en distintos puntos de interés en un sistema eléctrico, con lo cual se logra tener una perspectiva general del comportamiento de los parámetros eléctricos. De esta manera, se facilita la identificación del origen del fenómeno registrado, dado que se puede comprobar cómo fue su propagación en el sistema, estableciendo dónde tuvo un mayor impacto y de esta manera plantear posibles soluciones [1-3].

En este sentido, el artículo presenta los resultados del estudio de calidad de potencia realizado en un sistema industrial del sector hidrocarburos, en el cual se cuenta con gran cantidad de dispositivos que afectan o se ven afectados por los diversos fenómenos de calidad de potencia. Simultáneamente, se realiza el modelamiento y simulación del sistema con el fin de reproducir los fenómenos de calidad de potencia registrados y a partir de esto se proponen soluciones para mitigar los problemas producidos por dichos fenómenos.

\section{MATERIALES Y MÉTODOS}

En esta sección se presenta la metodología propuesta para el análisis integral de calidad de potencia de una instalación eléctrica. Inicialmente se mide la calidad de potencia en diferentes puntos de la instalación eléctrica industrial, para lo cual se requiere de múltiples equipos de medición para hacer los registros de manera simultánea y durante los tiempos establecidos en la norma NTC 5001 [4]. A partir de los resultados y análisis de dichas mediciones se determinan los principales fenómenos que afectan la instalación. Posteriormente, se simula la instalación y se reproducen las perturbaciones identificadas. Para esto se puede emplear cualquier software de simulación que permita reproducir de la manera más fiel posible el comportamiento de la red en estado estable y ante problemas de calidad de potencia. Mediante simulaciones se implementan posibles soluciones a los problemas identificados, con lo cual se reducen los costos de implementación y se puede mejorar la respuesta del sistema una vez dichas soluciones son 
puestas en operación. Finalmente se hace una evaluación económica de las soluciones propuestas para seleccionar la mejor desde el punto de vista técnico y económico.

En resumen, para el desarrollo integral de un estudio de calidad de potencia se deben llevar a cabo los siguientes pasos:

1) Identificación de la categoría del problema: determinar qué fenómenos se están presentando.

2) Caracterización del problema: con la ayuda de los registros de calidad, establecer las posibles causas y consecuencias. En esta etapa se implementa la medición multipunto; sus principales consideraciones se detallan más adelante.

3) Identificar rango de posibles soluciones: se definen equipos o técnicas, y en qué parte del sistema eléctrico se podrían implementar.

4) Evaluación técnica de las soluciones: con ayuda del modelado del sistema, se evalúan las alternativas.

5) Solución óptima: se hace una evaluación económica, ya que no siempre es posible implementar la mejor solución técnica por su elevado costo. Sin embargo, es importante aclarar que la evaluación económica no hacía parte del alcance del estudio presentado en este artículo.

\section{A. Consideraciones para realizar medición multipunto}

Las principales consideraciones para implementar la medición multipunto se establecen a continuación:

- Instalar medidores en el PCC (punto común de conexión), y en cada uno de los circuitos principales derivados. Esto es relevante en industrias con gran cantidad de alimentadores, con el fin de facilitar el análisis y encontrar rápidamente el origen de los eventos.

- Instalar medidores donde se tengan cargas o equipos sensibles a fenómenos de calidad.

- Si se han identificado problemas que se presentan en un circuito exclusivamente, no será necesario hacer mediciones en circuitos adicionales.

- Si existe un transformador previo a la alimentación del sistema eléctrico, será muy conveniente instalar un medidor en el lado de alimentación del operador de red.

- Si dentro de la industria se presentan varios niveles de tensión, es recomendable instalar medidores en cada nivel, para así establecer el posible origen y propagación de los eventos. 
- En cuanto al período de evaluación, la normativa y la literatura al respecto recomiendan una semana, aunque en ocasiones los eventos pueden presentarse de manera más o menos continua en este rango, por lo cual es importante el criterio y experiencia de quien desarrolle el estudio de calidad, el cual evaluará si los datos recolectados son o no suficientes.

- Garantizar que los equipos estén sincronizados bajo la misma referencia en tiempo (GPS), para garantizar que el análisis multipunto sea correcto.

- Se recomienda caracterizar los fenómenos relacionados con variaciones de tensión en valores por unidad, con el fin de comparar diferentes registros y así establecer su posible origen.

- El costo de instalar varios analizadores es quizá la mayor desventaja del método, pero se debe tener en cuenta que en determinadas situaciones la demora en encontrar la causa de los problemas de calidad puede traducirse en daño de equipos costosos y pérdidas en áreas productivas, cuyos costos pueden ser incluso más elevados que el de los mismos analizadores, por lo cual su implementación depende de las necesidades y tipos de equipos sensibles con los que cuenta la industria.

\section{RESULTADOS}

En esta sección se presentan los resultados de la evaluación de la calidad de potencia, siguiendo la metodología presentada en la sección anterior. Como caso de estudio se tiene un sistema que se alimenta a través de la red externa en $115 \mathrm{kV}$, la cual alimenta 5 circuitos en 34,5 kV a través del transformador principal de la industria (CPF 02 es respaldo del 01). El sistema es radial y, adicionalmente, cuenta con barrajes en 4,16 kV y $480 \mathrm{~V}$. La medición multipunto se realiza en $115 \mathrm{kV}$ y $34,5 \mathrm{kV}$, con el fin, no solo de identificar los fenómenos, sino de tratar de establecer su origen. La figura 1 muestra el diagrama unifilar general con la ubicación de los medidores disponibles para el estudio. No fue posible obtener las mediciones de los circuitos D y CPF01 debido a fallas en los equipos de registro.

Se analizaron cada uno de los fenómenos de calidad de potencia de acuerdo con los parámetros establecidos en la norma técnica colombiana NTC 5001 [4]. El período evaluado comprendió una semana. Como resultado de los análisis, se identificó que los hundimientos de tensión y armónicos de tensión son los eventos que más afectaron al sistema, por lo cual los análisis posteriores se centran en estos dos fenómenos. 


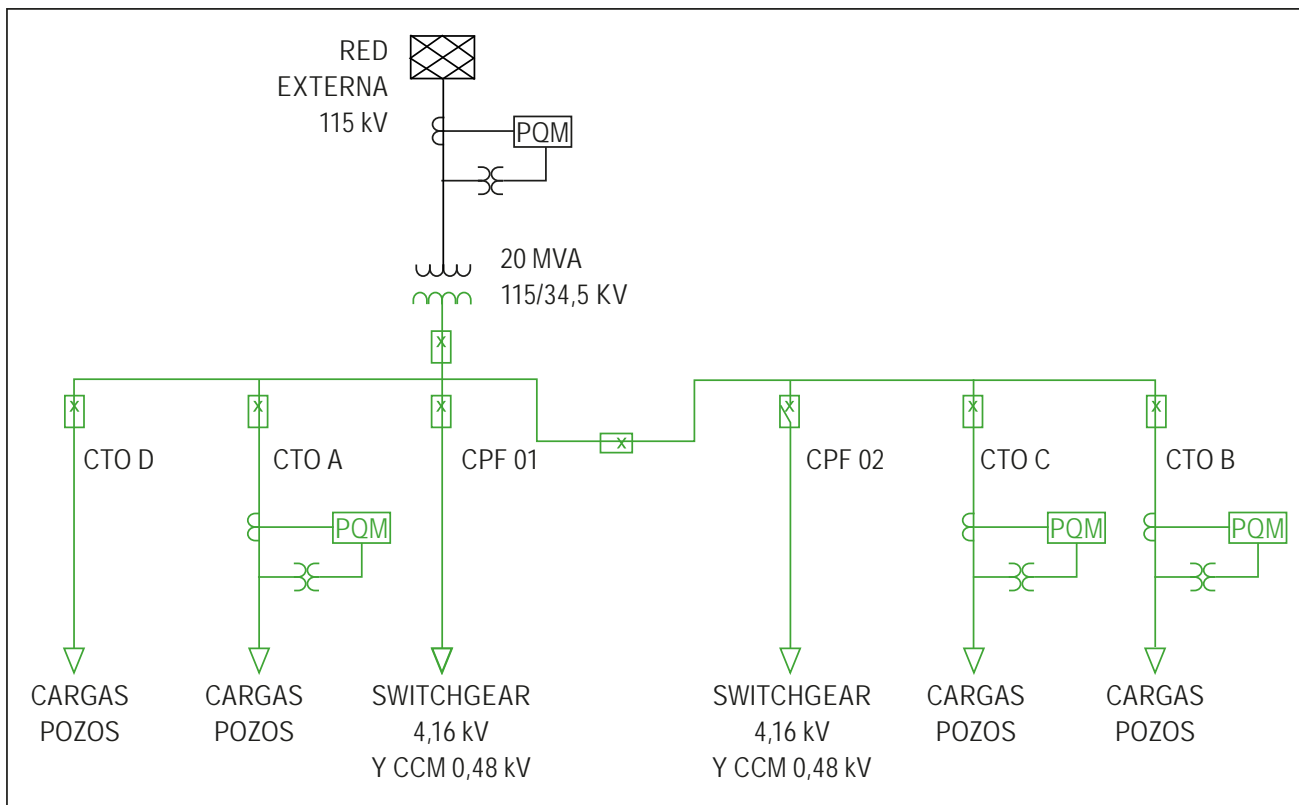

Figura 1. Diagrama unifilar general, y ubicación equipos de medición Fuente: elaboración propia

\section{A. Análisis de mediciones de hundimientos de tensión}

La figura 2 muestra un hundimiento registrado mediante la medición multipunto, tanto en la red externa (115 kV), como en uno de los circuitos de $34,5 \mathrm{kV}$ de la industria. De acuerdo con la tabla 1, el hundimiento que presenta mayor magnitud y duración es el registrado en la red externa (115 kV), por lo cual es posible establecer que es allí donde ocurrió el evento que ocasionó el hundimiento registrado en todo el sistema. Es importante resaltar que una sola medición no hubiera permitido hacer la caracterización y establecimiento del origen del evento con certeza.
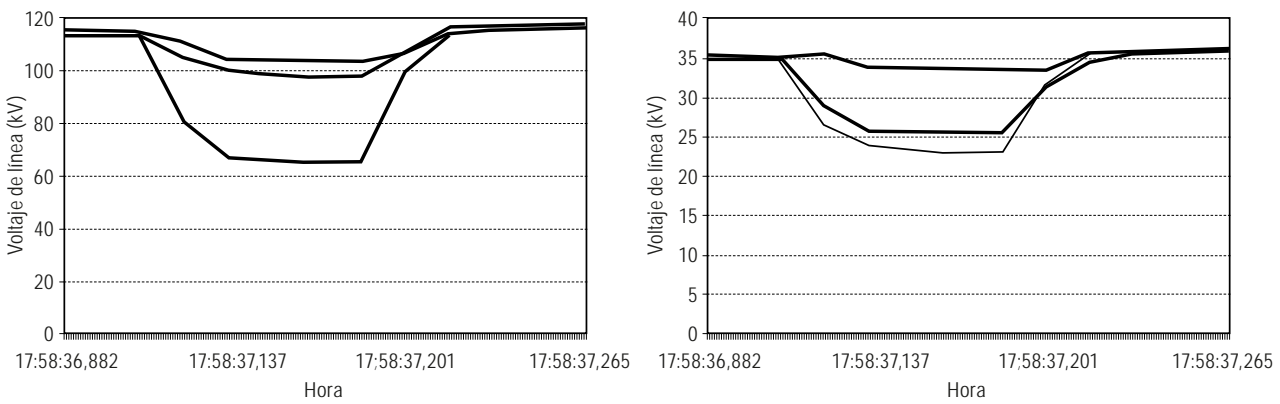

Figura 2. Hundimiento de tensión registrado en 115 kV y 34,5 kV. Tensiones de línea. 
Tabla 1. Caracterización hundimiento de tensión.

\begin{tabular}{lcccccc}
\hline \multicolumn{1}{c}{$\begin{array}{c}\text { Tensión nominal } \\
\quad(k V)\end{array}$} & $\begin{array}{c}\text { Duración } \\
(\mathrm{ms})\end{array}$ & \multicolumn{2}{c}{ Magnitud } & \multicolumn{2}{c}{ Corriente (A) } & Salida carga (\%) \\
115 & 98,99 & 65,04 & 0,565 & 33,1 & 20,0 & $39,6 \%$ \\
$\begin{array}{l}\text { Circuito A - } \\
34,5\end{array}$ & 91,55 & 22,04 & 0,667 & 15,0 & 14,9 & $0,7 \%$ \\
Circuito B - 34,5 & 93,50 & 22,94 & 0,665 & 19,8 & 18,5 & $6,6 \%$ \\
Circuito C - & 95,32 & 23,00 & 0,666 & 11,3 & 10,7 & $5,3 \%$ \\
34,5 & & & & & & \\
$\begin{array}{l}\text { Circuito D - } \\
34,5\end{array}$ & ND & ND & ND & ND & ND & ND \\
CPF01 & ND & ND & ND & ND & ND & ND \\
\hline
\end{tabular}

ND: No disponible

Además, se determinó que posiblemente la falla que se presentó en la red externa fue ocasionada por un evento que afectó 2 fases, la cual se propagó a la red de 34,5 kV, en donde afectó principalmente una fase [5-7]. Este hecho se confirma en la siguiente sección a partir de los resultados de las simulaciones.

Adicionalmente se analizaron los perfiles de corriente de los circuitos en los cuales fue posible efectuar mediciones, confirmando que el hundimiento desencadenó una salida de aproximadamente el 40\% de la carga en $115 \mathrm{kV}$. Sin embargo, en los circuitos de 34,5 kV de los que se tuvo registro no se evidenció una salida de carga considerable, por lo que se establece que esta salida se presentó en los otros circuitos de los que no se obtuvieron registros (D y CPF01).

\section{B. Análisis de mediciones de armónicos}

Como se puede apreciar en la figura 3, los niveles de distorsión armónica individual de tensión (Dv) existentes en los circuitos de 34,5 kV (industria) son inferiores al límite establecido por la norma de evaluación $(3 \%$ para $1 \mathrm{kV}<\mathrm{Vn} \leq 69 \mathrm{kV}, 1,5 \%$ para $69 \mathrm{kV}$ $<\mathrm{Vn} \leq 161 \mathrm{kV})$. En cuanto al circuito alimentador de $115 \mathrm{kV}$, superó el límite máximo de Dv establecido para este nivel de tensión en el quinto armónico. Igualmente, la distorsión armónica total de tensión (THDv) superó el límite permitido en la red externa $(2,5 \%$ en $115 \mathrm{kV})$.

A partir de las mediciones realizadas en $115 \mathrm{kV}$, se observa que la mayoría del tiempo la potencia armónica tiene valores positivos, indicando que la contaminación armónica proviene principalmente de la red externa hacia la industria [8]. 

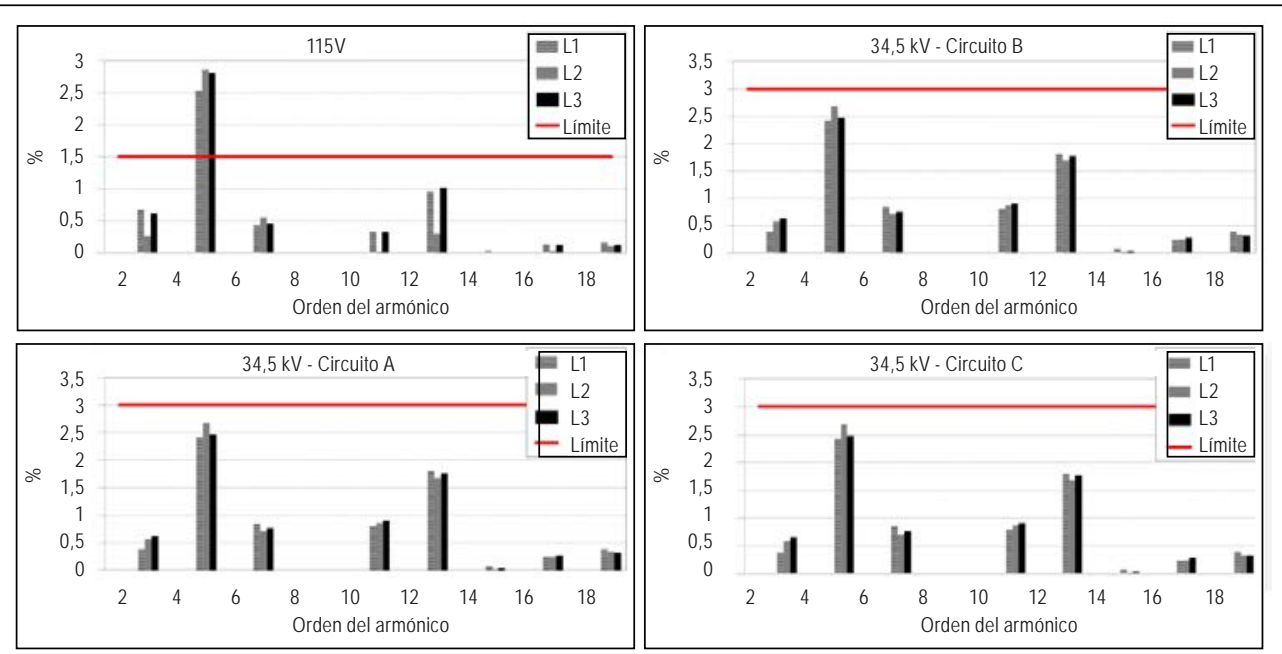

Figura 3. Distorsión armónica individual registrada en 115 kV y 34,5 kV.

Fuente: elaboración propia

\section{PROCESO DE SIMULACIONES Y DISCUSIÓN}

La principal restricción al momento de analizar la calidad de potencia en una instalación eléctrica es la cantidad limitada de información con la que se cuenta. Este problema se soluciona parcialmente mediante la medición multipunto; sin embargo, como se evidencia en este artículo, aun empleando esta técnica la cantidad de información de los fenómenos que pueden afectar a las redes eléctricas es bastante limitada. En tal sentido, como complemento a las mediciones, a continuación se presentan los resultados obtenidos mediante las simulaciones aplicadas en el programa Digsilent Power Factory. Estas simulaciones permiten tener un diagnóstico más completo de la instalación y probar y seleccionar los elementos requeridos para dar solución a los problemas identificados. Para este fin, primero se modeló el sistema y luego se ajustó al comportamiento real con ayuda de las mediciones, para así reproducir los eventos registrados. Posteriormente se identificaron y simularon las soluciones, comprobando su efectividad.

\section{A. Simulación de hundimientos de tensión}

De acuerdo con el análisis previo, se estableció que el hundimiento evaluado se originó en la red externa debido a una falla ocurrida en 2 fases. Esta falla se simuló y los resultados se observan en la figura 4. Esto se logró ajustando la impedancia de falla en la red externa hasta obtener valores similares a los registrados. El evento simulado presenta una diferencia en magnitud respecto al registro real de $2 \%$ en $115 \mathrm{kV}$ y de 
$0,3 \%$ en $34,5 \mathrm{kV}$. Mediante simulación fue posible confirmar que el evento se produjo en la red externa y que afectó dos fases.
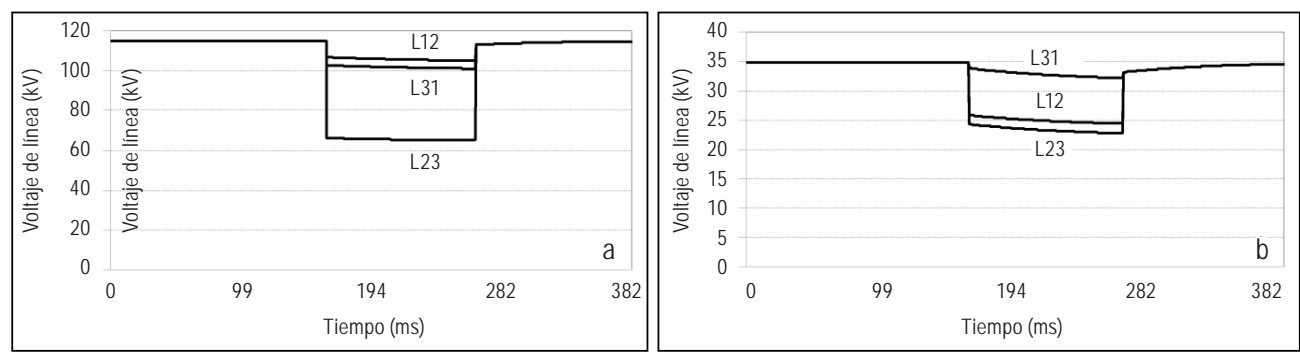

Figura 4. Simulación hundimiento de tensión en 115 kV y 34,5 kV

Fuente: elaboración propia

Luego, se utilizó el modelado para encontrar los parámetros eléctricos en puntos donde no se hicieron mediciones; en este caso se caracteriza el hundimiento en 4,16 $\mathrm{kV}$ y $480 \mathrm{~V}$. Mediante la simulación se comprobó la propagación de la falla en la industria. Mientras que en $115 \mathrm{kV}$ el hundimiento tuvo una magnitud de 0.56 p.u., en $480 \mathrm{~V}$ se alcanzó un valor de 0,74 p.u., y ya que allí se encuentra la mayoría de equipos electrónicos sensibles, en particular, variadores de frecuencia (VFD); es posible que la salida de carga se haya presentado como consecuencia del hundimiento en este nivel de tensión [9-12].

Como posible solución se determinó el uso de un TSC (Thyristor Switched Capacitor), que consta de un banco de condensadores conectado al sistema de potencia mediante tiristores, el cual se utiliza para compensación de potencia reactiva, o para el control dinámico de tensión [13, 14]. El diseño se basa en el conocimiento de la potencia reactiva y la caída de tensión durante el hundimiento [15], valores que fueron hallados con ayuda del modelo ajustado. Los principales parámetros de diseño son: potencia 15 MVAr, activado por tensión, límite superior de tensión 1,02 p.u., límite inferior de tensión 0,93 p.u. y tiempo de conmutación del tiristor 8,33 ms.

Se definió que el dispositivo se localizaría en $480 \mathrm{~V}$, donde se encuentran los equipos más sensibles ante hundimientos. El dispositivo se diseñó en un caso crítico de falla trifásica para compensar caídas de magnitud 0,7 p.u., debido a que al reproducir los eventos que se presentaron en alta tensión, se encontró en el simulador que esta sería la magnitud mínima en $480 \mathrm{~V}$. El TSC se diseñó para ser implementado en 5 etapas de 3 MVAr, con el fin de mejorar su respuesta dinámica $[14,16]$, dado que no todos los hundimientos tendrán la misma profundidad. Los resultados de simulación en $480 \mathrm{~V}$, antes y después de la implementación del TSC, se muestran en la tabla 2 y la figura 5. 
Tabla 2. Resultados de la implementación del TSC en 480 V.

\begin{tabular}{|l|c|c|}
\hline & Evento sin TSC & Evento con TSC \\
\hline Magnitud & 0,689 p.u. & 0,845 p.u. \\
\hline Duración & $90 \mathrm{~ms}$ & $17 \mathrm{~ms}$ \\
\hline
\end{tabular}

p. u.: valores en por unidad; ms: milisegundos

Fuente: elaboración propia.
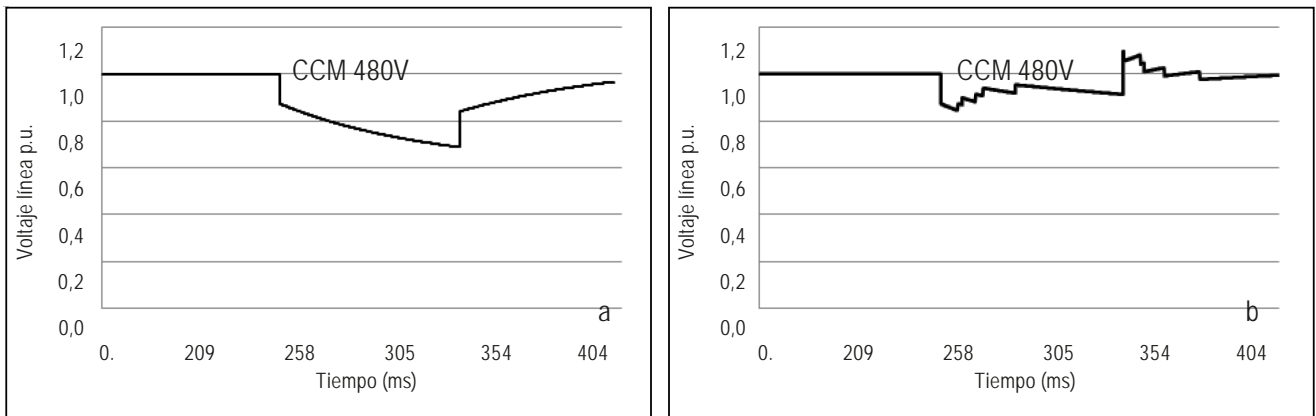

Figura 5. Simulación respuesta del TSC.

Se observa en la figura de la derecha la respuesta del TSC.

Fuente: elaboración propia

A partir de las simulaciones se pudo comprobar la efectividad del TSC en la disminución de la magnitud del hundimiento en 15,6\%, mientras que en duración se redujo en $73 \mathrm{~ms}$.

\section{B. Simulación de armónicos}

Para el modelado y simulación de armónicos se tuvo en cuenta lo siguiente:

1) Para reproducir la inyección de armónicos de los motores accionados por VFD se asumió un modelo de rectificador de seis pulsos, de acuerdo con la tabla 10-2 de la IEEE Std. 399 (1997) [17], dado que es el único modelo que considera el armónico individual $5^{\text {to }}$, presente en la industria.

2) La inyección de armónicos provenientes de la red externa fue modelada como una fuente de corriente, conforme a los valores medidos por el equipo registrador ubicado en $115 \mathrm{kV}$.

3) Se tuvieron en cuenta las consideraciones establecidas en la IEEE Std. 519 (1992) [18], para el modelado de los elementos del sistema industrial (motores, transformadores, conductores). 
De acuerdo con lo anterior, se recrearon los armónicos de corriente $5^{\text {to }}$ y $7^{\text {mo }}$, con el objetivo de reproducir los registros obtenidos mediante la medición multipunto. Luego, las simulaciones fueron empleadas para evaluar diferentes estrategias que permitan reducir la circulación de dichos armónicos por el sistema, a su vez, reduciendo las tensiones armónicas. La mayoría de las estrategias para mitigar armónicos están orientadas a implementar filtros de diferentes características y topologías. En este caso, se eligió un filtro paralelo sintonizado simple, dado que es rápido de implementar, filtra una armónica particular, y- la reducción en la circulación de un armónico de corriente representa una reducción en la caída de tensión armónica.

Los parámetros de diseño del filtro son [19-22]: $\mathrm{Q}=500 \mathrm{kVAr}, \mathrm{V}=38 \mathrm{kV}, \mathrm{C}=$ $920 \mathrm{pF}, \mathrm{L}=478,7 \mathrm{mH}, \mathrm{R}=24,06 \Omega$. Se seleccionó que el filtro se localizara en 34,5 kV para evitar la circulación del quinto armónico proveniente de 115 kV.

Se analizaron alternativas con frecuencias de sintonía cercanas al quinto armónico (300 Hz), pero bajo esta condición era posible que se presentaran problemas de resonancia. Aunque una frecuencia alejada del armónico a eliminar reduce su selectividad, garantiza que no se presente resonancia, por lo cual se selecciona una frecuencia de sintonía de $240 \mathrm{~Hz}$.

La figura 6 muestra la simulación de los armónicos $5^{\text {to }}$ y $7^{\text {mo }}$ con la implementación del filtro, logrando una reducción del $5^{\text {to }}$ armónico en $34,5 \mathrm{kV}$ de $0,45 \%$ en magnitud (10\%), mientras que en $115 \mathrm{kV}$ se logra una reducción del $5^{\text {to }}$ armónico de 0,2\% en magnitud (6\%).

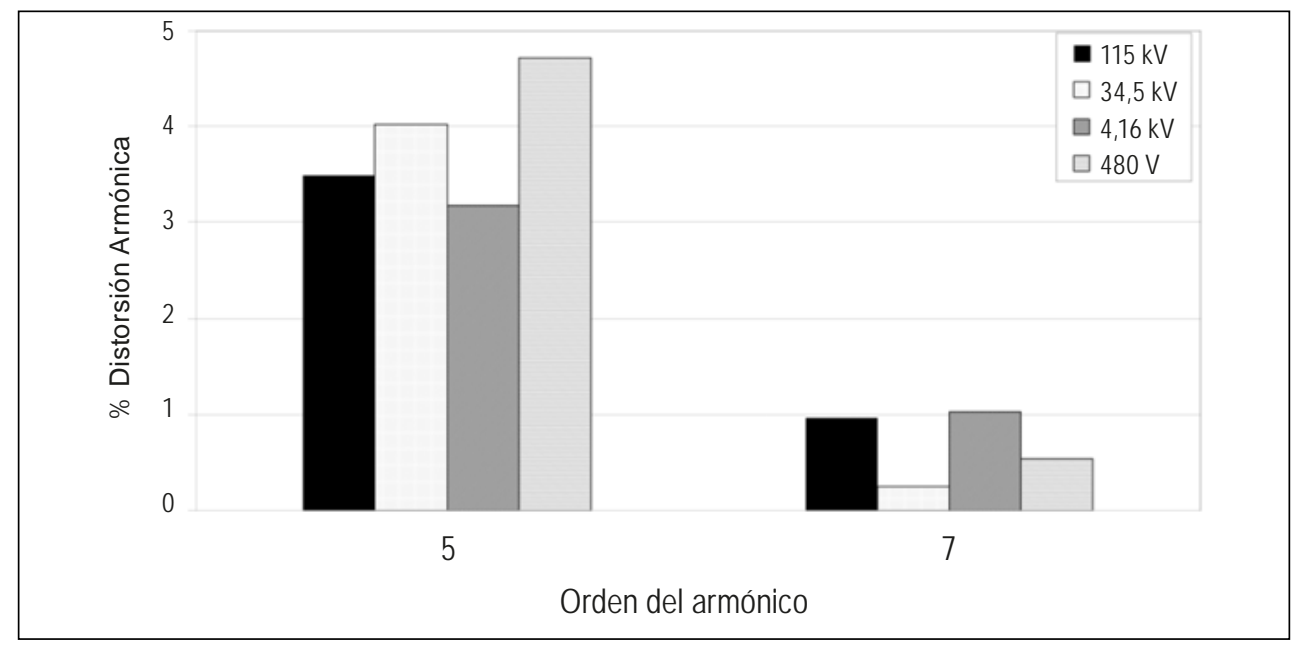

Figura 6. Reducción de $5^{\text {to }}$ armónico en $115 \mathrm{kV}$ y $34,5 \mathrm{kV}$ con la implementación del filtro Fuente: elaboración propia 


\section{CONCLUSIONES}

Se estableció una metodología general para adelantar estudios de calidad aplicando medición multipunto, y se definieron criterios particulares para aplicar esta medición en industrias o sistemas eléctricos similares.

Las mediciones multipunto permitieron analizar de manera global el origen y propagación de los eventos de calidad de potencia, hecho que no se hubiera podido lograr midiendo en un solo punto, lo cual permitió detectar los problemas de calidad de manera más rápida y certera.

Con la medición multipunto, se logró identificar el origen del hundimiento registrado, y su consecuencia en la producción de la industria. En cuanto a los armónicos, fue posible determinar que en su mayoría provienen del operador hacia la industria. Para ambos problemas fue posible simular y comprobar la efectividad de las soluciones propuestas con ayuda del software.

Se recomienda el uso de modelos en software de los sistemas bajo análisis de calidad de potencia; esto permitirá al analizador reproducir los eventos y comprobar la efectividad de las posibles soluciones, garantizando, además, que no introducirán problemas adicionales.

Los modelos de software ajustados con mediciones multipunto reales permiten conocer parámetros eléctricos adicionales en puntos donde no se realizaron mediciones, lo cual ayuda a hacer un análisis más profundo de la propagación e impacto de los eventos.

\section{REFERENCIAS}

[1] D. Castaldo, A. Ferrero, S. Salicone, and A. Testa, “A power-quality index based on multipoint measurements”, in 2003 IEEE Bologna Power Tech Conference Proceedings, 2003, vol. 4, pp. 722-726.

[2] D. Castaldo, D. Gallo, C. Landi, R. Langella, and A. Testa, "Power quality analysis: a distributed measurement system”, in 2003 IEEE Bologna Power Tech Conference Proceedings, 2003, vol. 3, pp. 487-492.

[3] C. Sankaran, Power Quality (Electric Power Engineering Series). Boca Ratón, Florida, 2002.

[4] Instituto Colombiano de Normas Técnicas, NTC 5001. Calidad de la potencia eléctrica, límites y metodología de evaluación en punto de conexión común. Colombia, 2008.

[5] Z. Klaic, K. Fekete, S. Nikolovski, and Z. Prekratic, "Propagation of the voltage sags through different winding connections of the transformers", 11th International Conference on Electrical Power Quality and Utilisation. pp. 1-5, 2011. 
[6] R. F. Mustapa, M. S. Serwan, N. Hamzah, and Z. Zakaria, "Effect of impedances line length to voltage sag propagation”, 2010 IEEE International Conference on Power and Energy. pp. 700-705, 2010.

[7] T. Sikorski and B. Solak, "Application of voltage and current transformations of different transformer winding connections in analysis of voltage dips propagation”, 2016 Electric Power Networks (EPNet). pp. 1-6, 2016.

[8] A. Baggini, Handbook of Power Quality. Bergamo, Italy: John Wiley \& Sons, Ltd., 2008.

[9] S. Kamble and C. Thorat, "Classification of voltage sags in distribution systems due to short circuit faults”, 2012 13th International Conference on Optimization of Electrical and Electronic Equipment (OPTIM). pp. 257-264, 2012.

[10] A. S. Poste, B. T. Deshmukh, and B. E. Kushare, "Detection, classification \& characterisation of voltage sag”, 2016 International Conference on Electrical, Electronics, and Optimization Techniques (ICEEOT). pp. 232-237, 2016.

[11] J. Caicedo, L. Navarro, E. Rivas, and F. Santamaria, "Voltage Sag Immunity Testing for Single-phase Electrical and Electronic Equipment", in VII Simposio Internacional sobre Calidad de la Energía Eléctrica (SICEL), 2013, pp. 1-6.

[12] L. F. Navarro, J. E. Caicedo, E. Rivas, and F. Santamaría, "Evaluación de la inmunidad de un motor de inducción monofásico frente a huecos de tensión”, Inf. tecnológica, vol. 25, no. 1, pp. 97-108, 2014.

[13] A. Ohtake, F. Zhang, T. Fujimoto, and N. Nakayama, "Development of 200-Mvar class thyristor switched capacitor supporting fault ride-through”, 2014 International Power Electronics Conference (IPEC-Hiroshima 2014 -ECCE ASIA). pp. 3857-3863, 2014.

[14] W. H. Ko and J. C. Gu, "Design and application of a thyristor switched capacitor bank for a high harmonic distortion and fast changing single-phase electric welding machine”, IET Power Electronics, vol. 9, n. ${ }^{\circ}$ 15. pp. 2751-2759, 2016.

[15] M. Alonso Martínez, "Gestión óptima de potencia reactiva en sistemas eléctricos con generación eólica (tesis doctoral)”, Universidad Carlos III de Madrid, España, 2010.

[16] S. Ghosh and M. H. Ali, "Power quality enhancement by coordinated operation of thyristor switched capacitor and optimal reclosing of circuit breakers”, IET Generation, Transmission \& Distribution, vol. 9, n. ${ }^{\circ}$ 12. pp. 1301-1307, 2015.

[17] I. of E. and E. Engineers, IEEE Std 399-1997. IEEE Recommended Practice for Industrial and Commercial Power Systems Analysis. USA, 1997, p. 488.

[18] I. of E. and E. Engineers, IEEE Std 519-1992. IEEE Recommended Practices and Requirements for Harmonic Control in Electrical Power Systems. USA, 1992, p. 112.

[19] M. D. Kusljevic, “A Simple Method for Design of Adaptive Filters for Sinusoidal Signals”, IEEE Trans. Instrum. Meas., vol. 57, no. 10, pp. 2242-2249, Oct. 2008. 
[20] T. Adrikowski, D. Buła, and M. Pasko, "Selection of method for reactive power compensation and harmonic filtering in industrial plant”, 2017 Progress in Applied Electrical Engineering (PAEE). pp. 1-5, 2017.

[21] J. Cheng, D. Chen, Y. Hu, and G. Chen, "An improved SHE algorithm and filter design method for high power grid-connected converter under unbalanced and harmonic distorted grid”, 2017 IEEE 26th International Symposium on Industrial Electronics (ISIE). pp. 594-599, 2017.

[22] J. Wang, M. Zhang, S. Li, T. Zhou, and H. Du, "Passive filter design with considering characteristic harmonics and harmonic resonance of electrified railway," $20178^{\text {th }}$ International Conference on Mechanical and Intelligent Manufacturing Technologies (ICMIMT). pp. 174-178, 2017. 